\title{
Mechanical And Thermodynamic Stabilities, Half- Metallic Property of Co2CrAl Heuslerene: A DFT Study
}

A. Boochani ( $\square$ arash_bch@yahoo.com )

Islamic Azad University

M. Asshabi

Razi University

M. Jamal

Islamic Azad University

M. Shahrokhi

Islamic Azad University

N. B. Nezafat

Institute for Research in Fundamental Sciences

N. Salimi

Islamic Azad University

M. Amiri

Islamic Azad University

\section{A. Yari}

Islamic Azad University

S. Solaymani

Islamic Azad University

F. Motamed Dezfuli

Islamic Azad University

S. Jalali Asad-Abadi

University of Isfahan (UI)

\section{A. Zelati}

Birjand University of Technology

N. A. Vahabzadeh

Parsabad Moghan Branch, Islamic Azad University

\section{Research Article}

Keywords: DFT, Co2CrAl Heuslerene, Elastic stability, Dynamic stability, Phase diagram, Electronic structure 
Posted Date: September 1st, 2021

DOI: https://doi.org/10.21203/rs.3.rs-778082/v1

License: (c) (i) This work is licensed under a Creative Commons Attribution 4.0 International License. Read Full License 


\title{
Mechanical and Thermodynamic Stabilities, Half-Metalic Property of Co2CrAl Heuslerene: A DFT Study
}

A. Boochani ${ }^{1, *}$, M. Asshabi ${ }^{2}$, M. Jamal ${ }^{1}$, M. Shahrokhi ${ }^{3}$, N. B. Nezafat ${ }^{4}$, N. Salimi ${ }^{5}$, M. Amiri ${ }^{1}$, A. Yari $^{1}$, S. Solaymani ${ }^{1,4}$, F. Motamed Dezfuli ${ }^{5}$, S. Jalali Asad-Abadi ${ }^{6}$, A. Zelati ${ }^{7}$, N. A. Vahabzadeh ${ }^{8}$

\author{
${ }^{1}$ Departement of Physics, Kermanshah Branch, Islamic Azad University, Kermanshah, Iran. \\ ${ }^{2}$ Department of Physics, Razi University, Kermanshah, Iran. \\ ${ }^{3}$ Young Researchers and Elite Club ,Kermanshah Branch, Islamic Azad University, Kermanshah, Iran. \\ ${ }^{4}$ School of Physics, Institute for Research in Fundamental Sciences, Tehran, Iran. \\ ${ }^{5}$ Department of Physics, Faculty of Sciences, Science and Research Branch, Islamic Azad University, Tehran, \\ Iran. \\ ${ }^{6}$ Department of Physics, University of Isfahan (UI), Hezar Gerib Avenue, Isfahan, 81746-73441, Iran. \\ ${ }^{7}$ Department of Basic Sciences, Birjand University of Technology, Birjand, Iran \\ ${ }^{8}$ Department of Physics, Parsabad Moghan Branch, Islamic Azad University, Parsabad Moghan, Iran.
}

\begin{abstract}
In the present study, the physical properties of the ground state in bulk and $\mathrm{Co}_{2} \mathrm{CrAl}$ Heuslerene compound are investigated by density functional theory (DFT). The effects of exchange-correlation potential on the calculations have been also investigated by GGA, GGA+U, and GGA+U+mBJ approximately. Here, three graphene-like structures with the thickness of about 8 Bohr have been labeled as $\alpha, \beta$, and $\Upsilon$ phases. The results demonstrate the mechanical stability of bulk $\mathrm{Co}_{2} \mathrm{CrAl}$ since it passes the elastic stability test. Having proved the static stability of the bulk and three Heuslerene shapes of $\mathrm{Co}_{2} \mathrm{CrAl}$, it is essential to study dynamic stability as well. The accessible region in the thermodynamic phase diagrams confirms the thermodynamic stability of bulk $\mathrm{Co}_{2} \mathrm{CrAl}$ and all $2 \mathrm{D}$ phases of the compound. According to our electronic calculations, the bulk phase of $\mathrm{Co}_{2} \mathrm{CrAl}$ is a half-metal whose values of magnetic moment and spin polarization is $3 \mu_{\mathrm{B}}$ and $100 \%$ at the Fermi level, respectively. Besides, $\alpha$ and $\Upsilon$ phases show the metal behavior for both spin directions in all imposed approximations. Finally, $\beta$ phase exhibits different magnetic properties for different approximations. From $3 \mathrm{eV}$ to $2 \mathrm{eV}$, GGA and $\mathrm{GGA}+\mathrm{U}$ reveal the magnetic anisotropic and isotropic nature. Besides, an extremely anisotropic nature is observed at the Fermi level by GGA+U+mbJ.
\end{abstract}

Key words: DFT, $\mathrm{Co}_{2} \mathrm{CrAl}$ Heuslerene, Elastic stability, Dynamic stability, Phase diagram, Electronic structure.

Corresponding author e-mail: arash_bch@yahoo.com 


\section{Introduction}

The unique properties of spin devices based on Heusler alloys motivated scientists to develop their fundamental knowledge about the application of these types of materials for industrial and research applications. Ferromagnetic materials with Heusler compounds base have significant applications in various modern technologies like data storage, sensors, and energy conversion. There are also some issues about ferromagnetic half Heusler and half-metallic (HM) alloys in the fields of solid state physics and material science $[1,2]$.

The synthesis of co-based Heusler compounds was begun in the 1970s [3]. Kübler et al. [4] found the disappearance of the minimum density of spin at Fermi energy $(E F)$ of $\mathrm{Co}_{2} \mathrm{MnSn}$ and $\mathrm{Co}_{2} \mathrm{MnAl}$ and which resulted in their specific transport properties because the majority of spin density contributes to $E F$ states. Meanwhile, de Groot et al. [5] introduced HM ferromagnets (HMFMs) which were expected to show $100 \%$ polarization of spin at $E F$. This made HMFMs ideal candidates for spintronic devices with spin injection [6]. The band structure (BS) must be calculated in details because of the important role of electronic structure in finding magnetic characterization of Heusler compounds and especially, predicting the HMFMs. The first attempts to calculate the BS of some Co-based Heusler compounds (Co2MnSn, Co2TiSi, and $\mathrm{Co} 2 \mathrm{TiAl}$ ) did not show HM ferromagnetism since the basis of calculations were spherical potentials. Moreover, for local spin density approximation (LSDA), simple form of the exchange-correlation potential was used [7-11].

Ishida et al. revealed the first signs of HM ferromagnetism in Co-based Heusler compounds for $\mathrm{Ru}_{2} \mathrm{MnC}$ and $\mathrm{Co}_{2} \mathrm{MnC}(C=\mathrm{Si}, \mathrm{Al}, \mathrm{Sb}$, and $\mathrm{Sn})$ [12, 13]. Mohn et al. [14] could only find the magnetic ground state of $\mathrm{Co}_{2} \mathrm{TiC}(C=\mathrm{Sn}$ and $\mathrm{Al})$ by full symmetry potentials, not the HM state. Galanakis et al. [15] also reported HM behavior in various $A_{2} B C$ compounds which was in good agreement with those found by Picozziet al for the Mn-based compounds. They used the generalized gradient approximation (GGA) because despite the pure LSDA function, the GGA technique considers the gradients of density [16]. Verifying the $\mathrm{HM}$ state of $\mathrm{Co}_{2} \mathrm{FeAl}$ is not possible by spherical potentials or GGA approximation and for the complete series of $\mathrm{Co}_{2} \mathrm{Cr}_{1-x} \mathrm{Fe}_{x} \mathrm{Al}$, the $\mathrm{HM}$ ferromagnetic ground state can be found when both GGA approximation and full symmetry potentials are applied $[17,18]$. This means that both of these parameters are essential for finding ground state and correct electronic structure of Heusler compounds.

The ferromagnetic properties with significant magnetic moment and high Curie temperature were observed in the majority of co-based full Heusler compounds with cubic L21 structure [19]. The Curie temperature and magnetic moment of $\mathrm{Co} 2 \mathrm{MnSi}$ are $985 \mathrm{~K}$ and $4.96 \mu \mathrm{B}$, respectively. Furthermore, many of them were 
predicted to be half-metal and hence, are of particular interest in spintronic devices $[20,21]$. Recenently, the electronic BS and magnetic properties of the full Heusler compounds have been investigated on a large scale, both theoretically and experimentally [19- 21]. For instance, the total magnetic moments in these compounds followed the Slater-Pauling-type behavior and were explained by their electronic structures [15]. Also, the Curie temperatures of Co-based Heulser compounds were calculated by $a b$ initio calculations related to the electronic structures [20].

The popularity of two-dimensional (2D) materials with the thickness of both single-atom and few- atoms was initiated from the discovery of graphene in 2004. These materials are considered as promising candidates for the next generation of ultrathin optoelectronics and electronics devices because of their mechanical, thermal, and electrical properties [21- 23]. Graphene is a single layer of $\mathrm{sp}^{2}$ bonded carbon atoms with high carrier mobility [24] which is almost chemically inert [25]. Its mechanical flexibility [26] makes it to be applied in flexible electronic and ultrathin 2D devices. Therefore, graphene has a wide variety of applications in transistors as well as chemical and strain sensors [27-28]. For group-IVA 2D materials like silicone, graphene, stanene, and germanene, the zero band gap can be opened by the adsorption of especial species or vertical electric field. However, their application in transistors is restricted at room temperature because in general, the value opened band gap is smaller less than $0.4 \mathrm{eV}[29,30]$. Recently, materials such as nitrogenene [31], black phosphorene (BP) [32], arsenene [33], and antimonene [34] in group-VA 2D have attracted great attention. The direct bandgap of monolayer and fewlayer BP is almost $0.59-1.51 \mathrm{eV}$ [35] and its unique electronic properties like ON/OFF current ratio and high carrier mobility $\left(\sim 1000 \mathrm{~cm}^{2} / \mathrm{Vs}\right)$ demonstrates its necessity in electronic and optoelectronic applications [36- 38]. The discovery of semiconductors and insulators has expanded 2D materials and graphene over the past few years $[39,40]$. Using 2D forms of the direct bandgap semiconductors in transition metal dichalcogenides (TMDs) like monolayer $\mathrm{WS}_{2}$ and $\mathrm{MoS}_{2}$ have significantly improved optoelectronics devices [41, 42]. However, further improvements require continuous investigations of new 2D materials. Scalable methods for creating 2D monolayers can not be used in bulk layered materials since their access to crystals has not been explored yet [43].

The high compactness and stability of thin films and 2D structures which are achieved by cutting the bulk form along crystallographic direction is due to the FCC structure of Heusler compounds in this direction [111]. In our last works, the graphene-like monolayers derived in this way have been subsumed under the new group of 2D materials, named Heuslerenes [44, 45]. As the DFT-based calculation approved, the two predicted Heuslerene forms of $\mathrm{Co}_{2} \mathrm{VAl}$ and $\mathrm{Ti}_{2} \mathrm{VGe}$ are statically and dynamically stable. Moreover, it was demonstrated 
that the combination of LDA and GGA+U approximations along with the $\mathrm{mBJ}$ exchange potential could modify the projection of the electronic maps of this group of materials and would reveal their HM characteristics. The main goal of the present work is to expand the idea of Heuslerenes as novel 2D structures by introducing a new member based on $\mathrm{Co}_{2} \mathrm{CrAl}$ and dealing with its expected properties in some detail.

\section{Computational methods}

The physical properties of the ground state of bulk and heuslerene of the $\mathrm{Co}_{2} \mathrm{CrAl}$ compound were studied based on density functional theory (DFT) by full potential linearized augmented plane waves (FP-LAPW) [46, 47]. Then, the effects of exchange-correlation potential on the calculations of electronic properties in the Wien2K [65] code were investigated by GGA [48], GGA+U [49-50], and GGA+U+mBJ [51, 52] approximations. The spin polarization calculations were also considered and the Hubbard potential (U) values applied to the $\mathrm{Co}$ and $\mathrm{Cr}$ atoms were about $0.235 \mathrm{Ry}$ and $0.182 \mathrm{Ry}$ respectively. The applied cut-off parameter $\left(\mathrm{R}_{\mathrm{mt}} \mathrm{K}_{\max }\right)$ was 8 and the Brillouin zone integration were about $17 \times 17 \times 17$ and $19 \times 19 \times 5$ kpoints according to the bulk and Heuslerene phase, respectively. The radius of the spheres in $\mathrm{Co}$ and $\mathrm{Cr}$ atoms were 2.15a.u and for Al 2.00 a.u, respectively. The crystal elastic constants for the bulk structure were obtained by using the method proposed by Jamal [55, 56] without considering the Hubbard $\mathrm{U}$ parameter $(\mathrm{GGA}+\mathrm{U})$ in the Wien $2 \mathrm{~K}$ framework. Also, calculating elastic parameters for the 2D systems were carried out via the methods that were previously used [47]. The crucial study of dynamic stability is because it provides insight into the possible distortions in the crystal. Thus, we have analyzed phonon dispersion curves by a Quantum Espresso package [53-54]. Also, vibrational frequencies and force constants are calculated by the linear response of density functional perturbation theory.

\section{Results}

\subsection{Stability of $\mathrm{Co}_{2} \mathrm{CrAl}$ and its 2D sheets}

$\mathrm{Co}_{2} \mathrm{CrAl}$ is a Co-based full-Heusler compound crystallized in the F.C.C. structure with the $\boldsymbol{F} \boldsymbol{m} \overline{\mathbf{3}} \boldsymbol{m}$ space group. As mentioned above, the films cut along its [111] crystallographic direction must be hexagonal, dense enough, and stable. Thus, by considering the atomic termination of the final slices, we have produced three ultrathin films of about 8 Bohr thick, named $\alpha, \beta$ and $\Upsilon$ phases (see Fig. 1). It is important to note that the stoichiometry of each product is different from the main source as $\mathrm{Co}_{2} \mathrm{CrAl}_{2}, \mathrm{Co}_{2} \mathrm{Cr}_{2} \mathrm{Al}$, and $\mathrm{Co}_{3} \mathrm{CrAl}$ for the $\alpha$, 
$\beta$, and $\Upsilon$ shapes, respectively. We will see that this extra atom in each monolayer plays a positive role in its stability.

In order to maintain a system, the first step is to find equilibrium volume at minimum energy. The total energy of each system has been calculated by fitting it to the Brich-Mornaghan equation for different unit cell volumes and Fig. 2 illustrates the final E-V plots. Fig. 2 (a)-(d) represent symmetric outline with a minimum point which confirm the static stability of the bulk and three Heuslerene forms of $\mathrm{Co}_{2} \mathrm{CrAl}$. Comparing Fig. 2 (a) and (b) illustrates similar shapes of the energy-volume curves of the bulk state and the alpha phase. Beta and gamma phases of the Heuslerene $\mathrm{Co}_{2} \mathrm{VAl}$ are also similar. In the alpha phase, the slope of the curve increases with decreasing volume (under stress) which indicates more resistivity of Heuslerene plate in this condition. In other two phases, the relative symmetry on both sides of the equilibrium point of the curves are observed.

The results of the energy-volume curves are listed in Table 1. It is shown that, the lattice constant obtained of $\mathrm{Co}_{2} \mathrm{VAl}$ is agreed with other reported. The magnetic moment of this compound is integer 3, which is in accordance with Slater- Pauli rule, which indicates that this compound is a suitable option for spintronic purposes. In Table 1 , the $\mathrm{Co}_{2} \mathrm{VAl}$ bulk modulus indicates that the hardness of this crystal is twice that of steel metal, therefore, this compound has the necessary resistance for use in industrial tools and parts. The derivative of bulk modulus coefficient indicates that the bonds between its atoms are ionic type, which results in a high crystalline strength. The negative values of formation energy for the bulk phase and the Heuslerene show that they are all quite stable from an energy point of view. The lattice constants of the Heuslerenes are very close to each other in all three phases, indicating their structural similarity to each other, and as shown in Fig. 1, they differ in the arrangement of the atoms. The $\mathrm{Co}_{2} \mathrm{VAl}-\beta$ Heuslerene magnetic moment is an integer 1, indicating that the compound could be used in the spintronic industry. The bulk modulus of these two-dimensional structures show, that due to their low thickness (a few atomic layers) they have a suitable hardness. Their derivative of bulk modulus also testifies to the existence of strong ionic bonds between their atoms. 

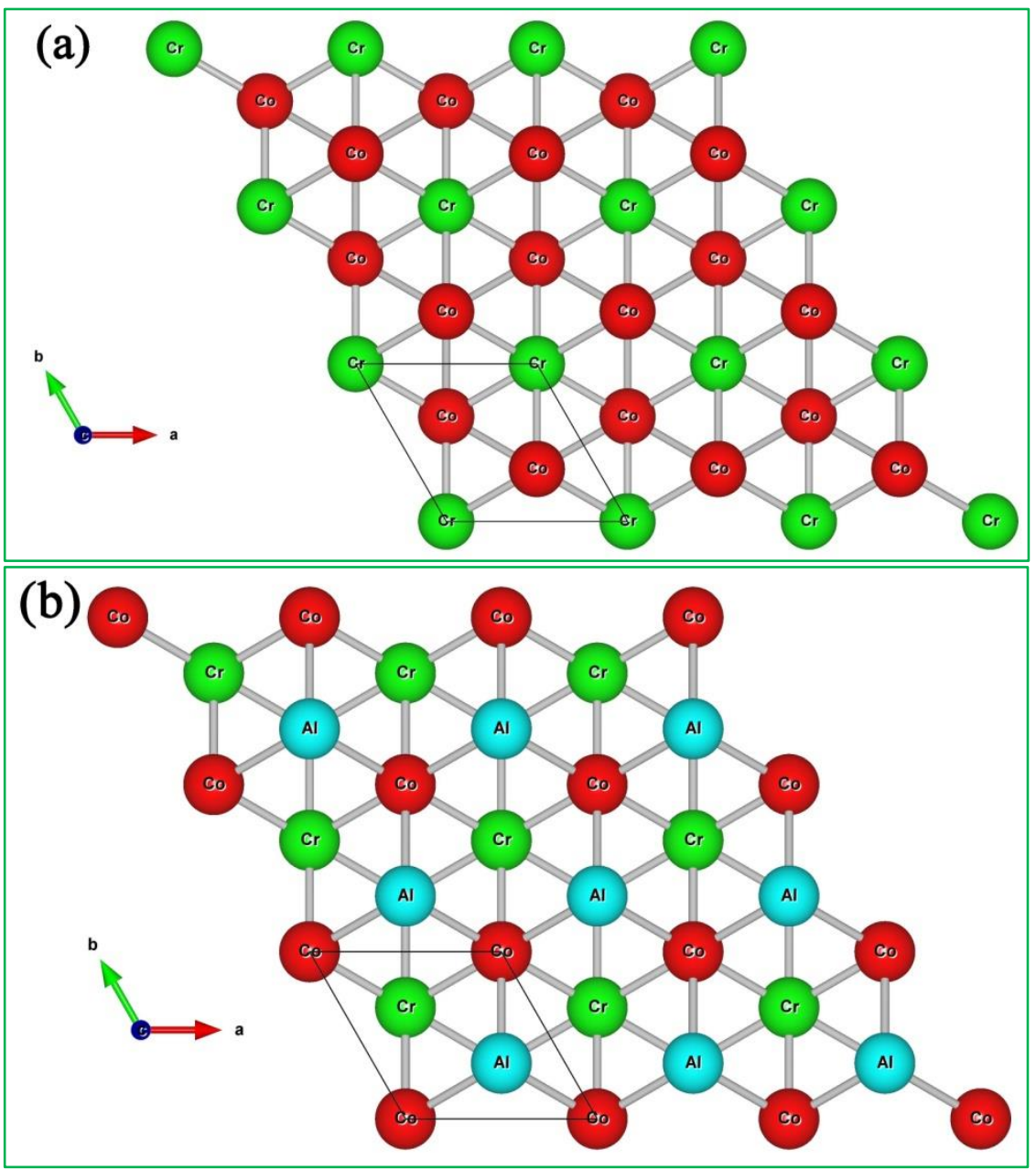


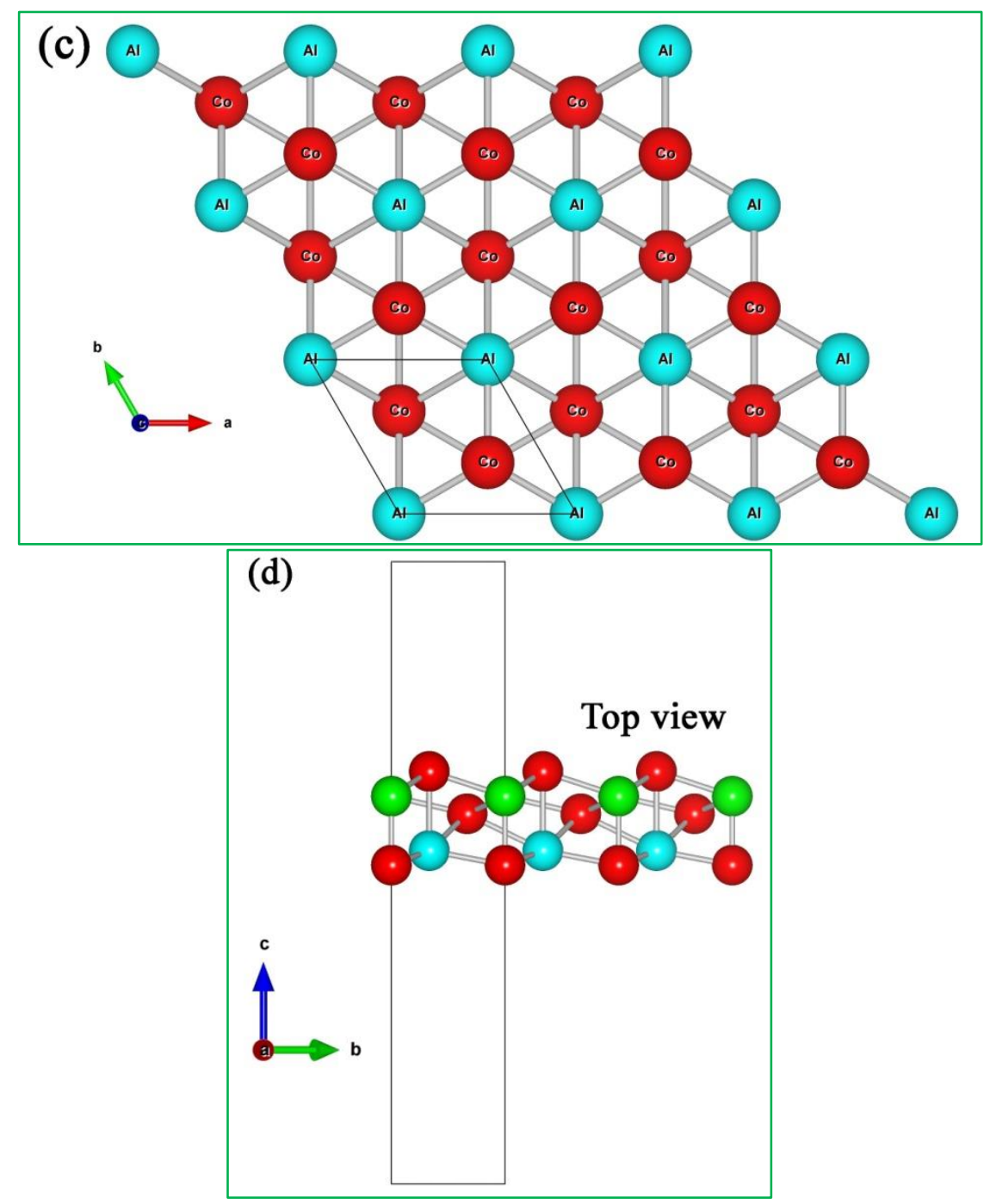

Fig.1. Crystal structure from top and side view; (a) $\alpha-$, (b) $\beta$ - and, (c) $\gamma$-phase

Table:1 Physical and mechanical properties of $\mathrm{Co}_{2} \mathrm{CrAl}$ compound in bulk and three Heuslerene phases, include; crystal lattice $(\mathrm{a}, \mathrm{c})$, formation energy $\left(\mathrm{E}_{\mathrm{f}}\right)$, total magnetic moment $\left(\mathrm{M}_{\mathrm{tot}}\right)$, Bulk modulus $(\mathrm{B})$, and derivative bulk modulus (B').

\begin{tabular}{lllllcc}
\hline Compound & $a_{0}\left(\mathrm{~A}^{\circ}\right)$ & $\mathrm{c} / \mathrm{a}\left({ }^{\circ} \mathrm{A}\right)$ & $\mathrm{E}_{\mathrm{f}}(\mathrm{eV})$ & $\mathrm{M}_{\mathrm{tot}}\left(\mu_{\mathrm{B}}\right)$ & $\mathrm{B}(\mathrm{GPa})$ & $\mathrm{B}^{\prime}$ \\
\hline $\mathrm{Co}_{2} \mathrm{CrAl}$ & 5.70 & 1.0 & -0.3773 & 3.00 & 204.9 & 5.00 \\
$\alpha$-phase & 7.15 & 3.75 & -0.1858 & 0.00 & 39.3 & 4.50 \\
$\beta$-phase & 7.54 & 3.75 & -01742 & 1.00 & 41.6 & 4.51 \\
$\gamma$-phase & 7.50 & 3.75 & -0.1750 & 7.15 & 38.6 & 4.53 \\
& & & & & & \\
\hline
\end{tabular}



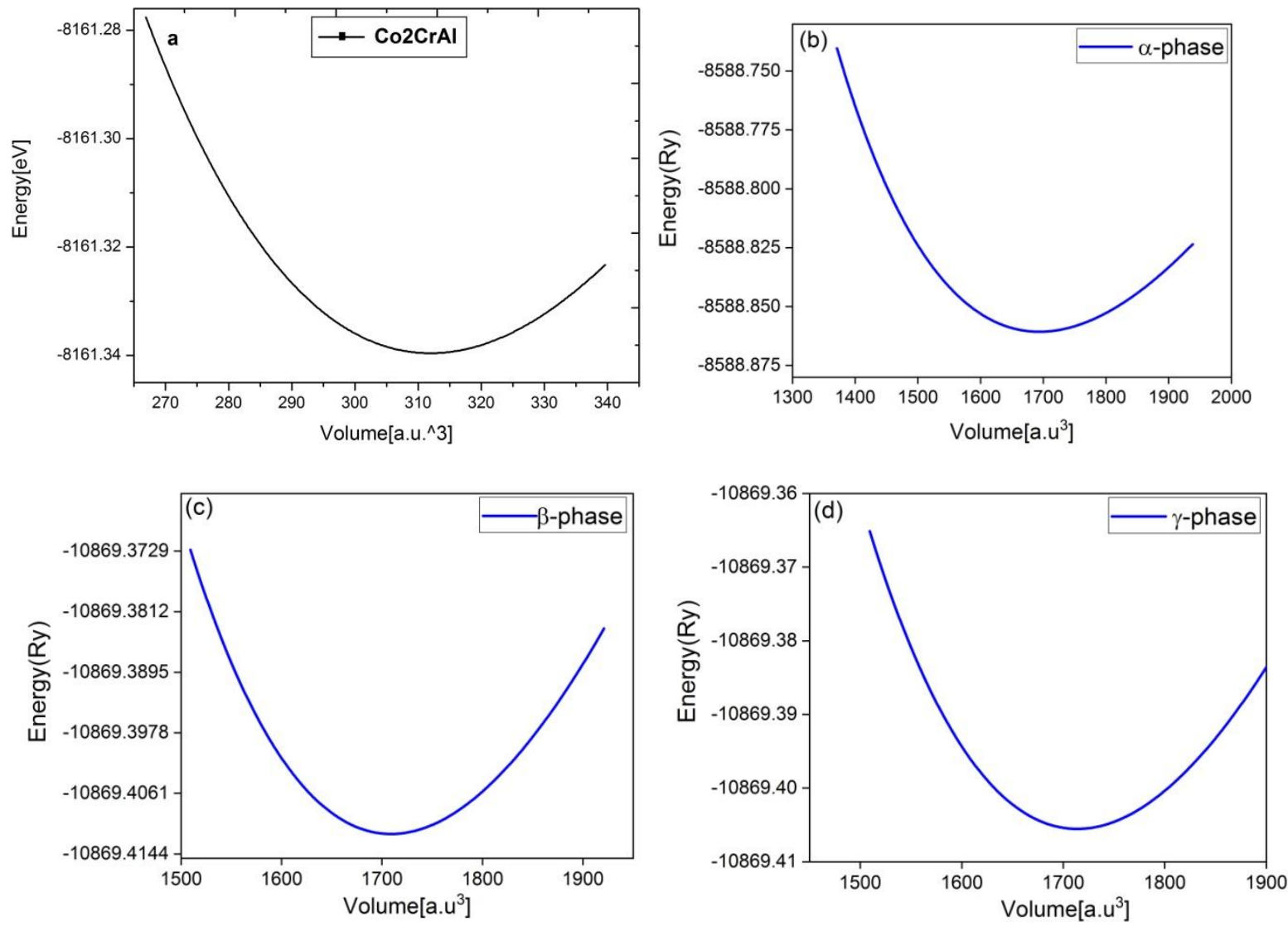

Fig. 2. E-V curves of (a) the bulk, (b) $\alpha$, (c) $\beta$ and (d) $\Upsilon$ Heuslerene phases of $\mathrm{Co}_{2} \mathrm{CrAl}$. Minimum points approve that all structures are statically stable.

\subsubsection{Mechanical stabilities}

\subsubsection{Bulk phase}

Elasticity refers to the retrieval of the original shape of materials after removing applied force. Hence, the amount of elastic constants can specify structural stability and can create a connection between the atomic and large scale in order to predict important parameters such as hardness, brittleness/ductility nature, melting temperature, type of bonds, stiffness, and etc [61]. These parameters can provide valuable information about the practical applications.

The theoretical background of the elastic constants along with the calculation of parameters such as bulk modulus, Shear modulus, Debye temperature, and ... for bulk materials with cubic symmetry have been reported in our previous reports [62, 63]. Moreover, as represented in our recent work [47], the theoretical background of the elastic constants for 2D materials as well as their appropriate amounts for the Heuslerene $\mathrm{Ti}_{2} \mathrm{VGe}$ were investigated by using IRelast2D package [64] which worked as an interface with Wien2k code [65]. It should be noted that the experimental investigation of the elastic constants of $2 \mathrm{D}$ materials is a challenge for scientists at present [66] and so, such a package 
(IRelast2D) might be the best alternative which opens a new theoretical way for investigating the mechanical properties of $2 \mathrm{D}$ systems.

Mechanical parameters revealed the fact that when the percentage of $\mathrm{B} / \mathrm{S}$ ratio of bulk material is less/more than $175 \%$, it is brittle/ductile [67]. The Cauchy pressure sign is defined as $\left(\mathrm{C}_{12}-\mathrm{C}_{44}\right)$ and specifies the sorts of bonds. Commonly, the dominance of covalent and ionic bonds specifies the sign of Cauchy pressure bulk materials to be negative or positive, respectively [68]. Moreover, Poisson's ratio determines the bond types so that for materials with covalent and with ionic bonds it is less than 0.25 and close to 0.25 or more, respectively [62].

Stiffness is the resistivity of the matter in reaction to an applied force so that a stiff material has a higher Young's modulus and a flexible material has less stiffness. Hence, higher values of Young's modulus are essential when deflection is disagreeable. Hardness, as another important parameter, indicates the resistivity of the matter to the shape variations. Among three parameters that indicate hardness i. e. shear modulus, bulk modulus, and Vickers hardness, better results can be achieved by characterizing the Vickers hardness [69] which can be calculated by the following equations provided by Chen and Tian:

$$
\begin{aligned}
& H_{V}^{\text {Chen }}=2\left(k^{2} S\right)^{0.585}-3 \quad\left(k=\frac{S}{B}, \text { S: shear , B: bulk modulus }\right) \\
& H_{V}^{\text {Tian }}=0.92 k^{1.137} S^{0.708}
\end{aligned}
$$

In the case of some ionic compounds in which Chen's equation detects negative values, Tian's equation reformulates it to solve this problem [70]. To evaluate thermodynamic parameters like Debye temperature $\left(\theta_{\mathrm{D}}\right)$ as well as transversal $\left(\mathrm{V}_{\mathrm{t}}\right)$, longitudinal $\left(\mathrm{V}_{\mathrm{l}}\right)$, average $\left(\mathrm{V}_{\mathrm{m}}\right)$ sound velocities. The Hill scheme is applied as an intermediate to the Voigt and Reuss [61]. Table 2 illustrates the elastic constants and mechanical and thermodynamic parameters of the bulk $\mathrm{Co}_{2} \mathrm{CrAl}$ as well as their comparison with the previous theoretical reports [71, 72] using the same methods and exchange-correlation (FP-LAPW-PBE) $[73,74]$. Our $a b$ initio results are in agreement with previous theoretical calculations. The value of Poisson's ratio implies that the domain bonds are ionic which is compatible with our results from the positive sign of Cauchy pressure. The percentage of $\left(\frac{B_{H}}{S_{H}}\right)$ ratio of bulk $\mathrm{Co}_{2} \mathrm{CrAl}$ is $214 \%$ from which the ductile nature is inferred. The values of Vickers Hardness, based on Chen's and Tian's equations, are positive and almost close to each other. For a crystal, the elastic stability criterion (known as Born stability conditions) emerges when the elastic energy of our compound is positive. From the mathematical point of view, it is held when eigenvalues of the elastic constants matrix $(\boldsymbol{C})$ are positive $[75,76]$. For cubic symmetries, we have: 


$$
C_{11}-C_{12}>0, C_{11}+2 C_{12}>0, C_{44}>0
$$

Our results indicate that bulk $\mathrm{Co}_{2} \mathrm{CrAl}$ is mechanically stable and passes the elastic stability test.

\subsubsection{Heuslerene phase}

The mechanical behavior of materials depends on their dimension and geometry [77-83]. This means that the physical behavior changes when we move from the bulk scale towards two- or one-dimensional scales. Table 3 represents the results gained by the IRelast2D package and PBE exchange-correlation for the elastic constants $\left(C_{i j}\right)$, shear moduli $(S)$, Young's moduli $(Y)$, and Poisson's ratios ( $v$ ) of the $\mathrm{Co}_{2} \mathrm{CrAl}-\mathrm{X}$ Heuslerenes where $\mathrm{X}=\{\mathrm{Al}$ ( $\alpha$-phase), $\mathrm{Cr}(\beta$-phase), $\mathrm{Co}(\gamma$-phase $)\}$ stands for the extra atom and the results are compared to the bulk stochiometry which resides at $(0,0,0)$ of the surface structure shown in Fig. 1.

We should recall that, as far as we know, no experimental or theoretical reports have been published about the $2 \mathrm{D} \mathrm{Co}_{2} \mathrm{CrAl}$ yet. The directional values of Young's moduli in Table 3 show that three Heuslerene phases of $\mathrm{Co}_{2} \mathrm{CrAl}$ are isotropic along the $\boldsymbol{x}$ and $\boldsymbol{y}$ directions. Furthermore, $\mathrm{Co}_{2} \mathrm{CrAl}-\mathrm{Al}$ ( $\alpha$-phase) has greater amounts of Young's moduli and thus, is stiffer than $\mathrm{Co}_{2} \mathrm{CrAl}-\mathrm{Cr}(\beta$ phase) and $\mathrm{Co}_{2} \mathrm{CrAl}-\mathrm{Co}(\gamma$-phase $)$ while it is of less stiffness than bulk shape. Shear modulus (shear stress/shear strain) which is the representative of hardness describes the resistance of the material to the shape changes. Hence, $\mathrm{Co}_{2} \mathrm{CrAl}$ $\mathrm{Al}$ is harder than two other Heuslerenes. The hardness decreases when we move from the bulk towards the surface.

As another result, all Poisson's ratios of the Heuslerenes are positive and independent of the $\boldsymbol{x}$ and $\boldsymbol{y}$ directions. The largest value belongs to $\mathrm{Co}_{2} \mathrm{CrAl}-\mathrm{Co}$ phase. As can be seen in Table 3, in $\mathrm{Co}_{2} \mathrm{CrAl}-\mathrm{X}$ Heuslerenes $(\mathrm{X}=\mathrm{Al}, \mathrm{Cr}, \mathrm{Co})$, the elastic parameters including $C_{11}, C_{22}, C_{66}, Y_{x}, Y_{y}$, and $S_{x y}$ decrease monotonically within $\mathrm{PBE}$ exchange- correlation while Poisson's ratio has an inverse behavior. $\mathrm{Co}_{2} \mathrm{CrAl}-\mathrm{Al}$ is mechanically stronger than other $\mathrm{Co}_{2} \mathrm{CrAl}$ Heuslerene phases since its elastic constants $\left(C_{11}\right.$ and $C_{12}$ and $\left.C_{66}\right)$ are larger than other phases. From the viewpoint of elasticity, all phases pass the elastic stability test and hence, they are mechanically stable. 
Table 1: The values of elastic constants $\left(\mathrm{C}_{\mathrm{ij}}\right)$, Bulk modulus $(\mathrm{B})$, Shear moduli (S) and Young's moduli (Y) in Voigt (V)-Reuss (R)-Hill (H) approximation in GPa, Poisson's ratios $(v)$, as well as transverse $\left(\mathrm{V}_{\mathrm{t}}\right)$, longitudinal $\left(\mathrm{V}_{\mathrm{L}}\right)$ and average wave velocities $\left(\mathrm{V}_{\mathrm{m}}\right)$ in $\mathrm{m} / \mathrm{s}$, Debye temperature $\left(\Theta_{\mathrm{D}}\right.$ in $\left.\mathrm{K}\right)$ and Vickers hardness base on the Chen and Tian equations $\left(\mathrm{H}_{\mathrm{V}}\right.$ in $\left.\mathrm{GPa}\right)$ for the bulk form of $\mathrm{Co}_{2} \mathrm{CrAl}$.

\begin{tabular}{|c|c|c|c|}
\hline$C U B I C$ & $\mathrm{Co}_{2} \mathrm{CrAl}$ & $\mathrm{Co}_{2} \mathrm{CrAl}^{\mathrm{a}}$ & $\mathrm{Co}_{2} \mathrm{CrAl}^{\mathrm{b}}$ \\
\hline$C_{11}$ & 274.85 & 265.0 & 278.07 \\
\hline$C_{12}$ & 180.12 & 196.2 & 187.94 \\
\hline$C_{44}$ & 161.69 & 156.1 & 151.91 \\
\hline$B_{V}$ & 211.70 & 201.5 & \\
\hline$B_{R}$ & 211.70 & 201.5 & \\
\hline$B_{H}$ & 211.70 & 201.5 & \\
\hline$S_{V}$ & 115.96 & & \\
\hline$S_{R}$ & 82.26 & & \\
\hline$S_{H}$ & 99.11 & 97.2 & \\
\hline$Y_{V}$ & 294.17 & & 280.66 \\
\hline$Y_{R}$ & 218.49 & & 208.98 \\
\hline$Y_{H}$ & 257.20 & 251.3 & 245.57 \\
\hline$v_{V}$ & 0.268 & & \\
\hline$v_{R}$ & 0.327 & & \\
\hline$v_{H}$ & 0.297 & 0.29 & \\
\hline$V_{T}$ & 3743.71 & & \\
\hline$V_{L}$ & 6973.03 & & \\
\hline$V_{m}$ & 4180.32 & & \\
\hline$\Theta_{D}$ & 550.50 & & \\
\hline$H_{V}^{\text {Chen }}$ & 9.11 & & \\
\hline$H_{V}^{\text {Tian }}$ & 10.05 & & \\
\hline
\end{tabular}

Previous theoretical results are bold.

${ }^{\mathrm{a}}$ :Ref. [71] (PBE). ${ }^{\mathrm{b}}$ :Ref. [72] (PBE). 
Table 3: The values of elastic constants $\left(C_{i j}\right.$ in $\left.\mathrm{N} / \mathrm{m}\right)$, Shear modulus $(S)$, and Young's modulus $(Y)$ in $\mathrm{N} / \mathrm{m}$ and Poisson's ratio ( $v$ ) for $\mathrm{Co}_{2} \mathrm{CrAl}-\mathrm{X}(\mathrm{X}=\mathrm{Al}, \mathrm{Cr}, \mathrm{Co})$ Heuslerenes.

\begin{tabular}{|c|c|c|c|c|c|c|c|c|c|c|}
\hline material & $C_{11}$ & $C_{22}$ & $C_{12}$ & $C_{66}$ & $Y_{x}$ & $Y_{y}$ & $S_{x y}$ & $v_{x y}$ & $v_{y x}$ & $\begin{array}{c}\text { Elastically } \\
\text { stable }\end{array}$ \\
\hline$\alpha$-phase & $\mathbf{1 2 0 . 3 6}$ & $\mathbf{1 2 0 . 3 6}$ & $\mathbf{5 2 . 3 6}$ & $\mathbf{3 3 . 9 2}$ & $\mathbf{9 7 . 5 5}$ & $\mathbf{9 7 . 5 5}$ & $\mathbf{3 3 . 9 9}$ & $\mathbf{0 . 4 4}$ & $\mathbf{0 . 4 4}$ & $\checkmark$ \\
\hline$\beta$-phase & $\mathbf{9 7 . 3 0}$ & $\mathbf{9 7 . 3 0}$ & $\mathbf{4 7 . 6 5}$ & $\mathbf{2 4 . 8 3}$ & $\mathbf{7 3 . 9 7}$ & $\mathbf{7 3 . 9 7}$ & $\mathbf{2 4 . 8 3}$ & $\mathbf{0 . 4 9}$ & $\mathbf{0 . 4 9}$ & $\checkmark$ \\
\hline$\gamma$-phase & $\mathbf{9 5 . 5 2}$ & $\mathbf{9 5 . 5 2}$ & $\mathbf{4 8 . 8 6}$ & $\mathbf{2 3 . 3 3}$ & $\mathbf{7 0 . 5 2}$ & $\mathbf{7 0 . 5 2}$ & $\mathbf{2 3 . 3 3}$ & $\mathbf{0 . 5 1}$ & $\mathbf{0 . 5 1}$ & $\checkmark$ \\
\hline
\end{tabular}

\subsubsection{Phonon distributions}

Having proved the static stability of the bulk and three Heuslerene shapes of Co2CrAl, it is essential to investigate their dynamical stability as well. For this aim, the phonon BSs in Fig. 3 (a-d) which concerns the crystal vibrations over the first Brillouin zone is studied. All frequency branches in these figures are positive implying that all discussed phases of $\mathrm{Co} 2 \mathrm{CrAl}$ are dynamically stable. For the bulk phase, steeper acoustical branches around $\Gamma$ point infer the transformation of thermal energy in $0-200 \mathrm{~nm}^{-1}$ region, and so, it may act as a good heat conductor along this direction. However, in the range of $200-270 \mathrm{~cm}^{-1}$ and $310-390 \mathrm{~cm}^{-1}$ two big optical gaps are observed and their optical branches have low slope and are nearly flat so that the IR energy with low mobility accumulates in this region.

Moreover, Fig. 3 (b-d) shows that all three 2D predicted forms of $\mathrm{Co}_{2} \mathrm{CrAl}$ are strongly stable against the lattice vibrations. These figures are plotted along the $\Gamma-\mathrm{K}-\mathrm{M}-\Gamma$ path within the first Brillouin zone. The acoustical branches of these Heuslerenes have a higher slope than optical branches. This can particularly observed in the $\alpha$ case where the slope of acoustical branches around the $\Gamma$ point is more and thus, this phase can better convey the low-frequency thermal energies. Three phonon band gaps for the $\beta$ case and one phonon band gap in the $\Upsilon$ case are observed frequencies more than $240 \mathrm{~cm}^{-1}$ and in the range of 340$400 \mathrm{~cm}^{-1}$, respectively. Because of qualitative similarity in the phonon BSs (such as two optical gaps), more similarity is expected in optical behavior of the $\beta$ phase as compared to the bulk. In addition, comparing the top energy levels of these $2 \mathrm{D}$ structures confirms its highest amount $\left(400 \mathrm{~cm}^{-1}\right)$ in $\Upsilon$ phase which is still a bit lower than that of the bulk shape. This means that any change in the type and arrangement of the comprising atoms may result in changes in the energy levels and optical behavior (including IR conductivity) of the ultimate compound. 

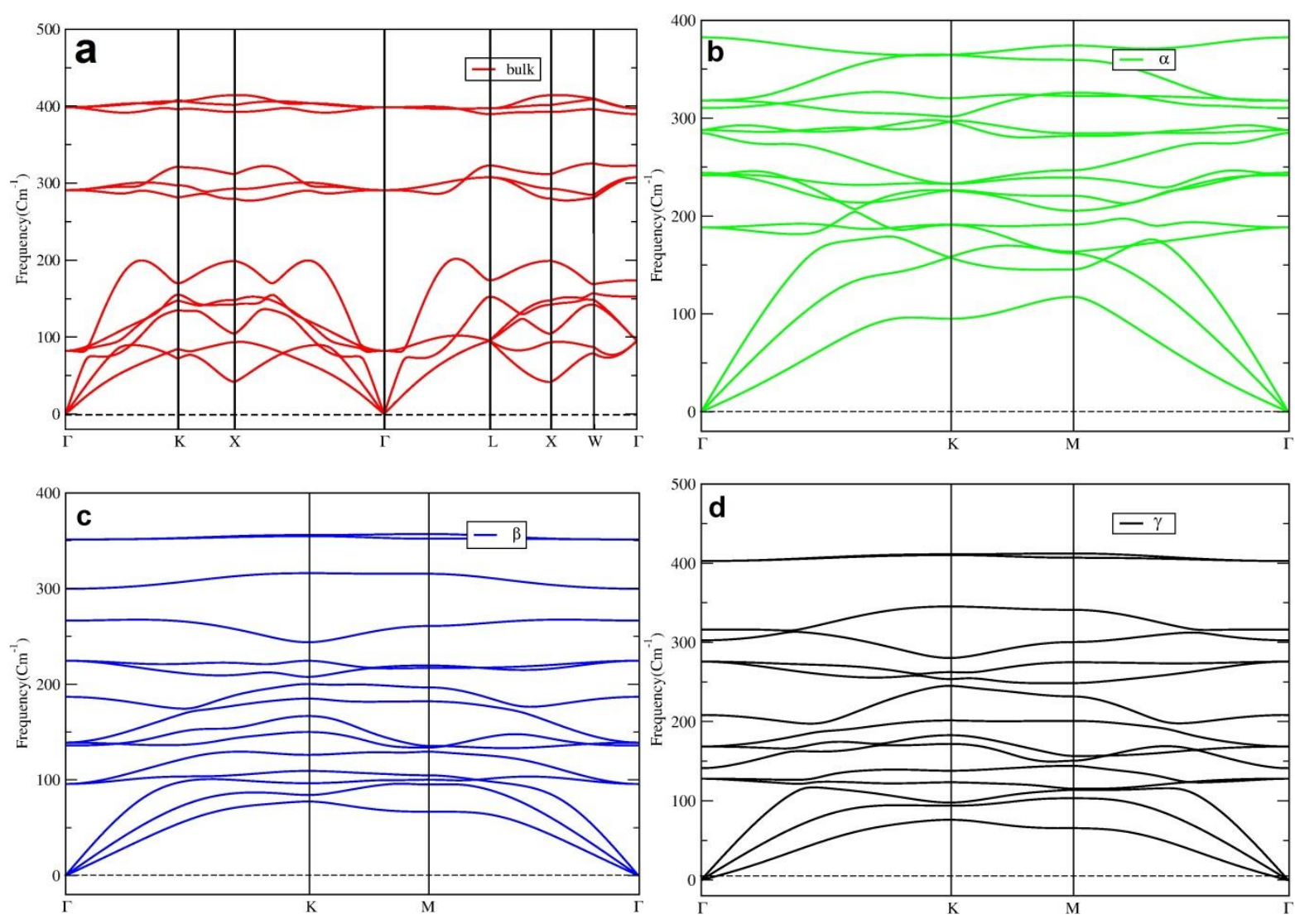

Fig. 3. Phonon BS of $\mathrm{Co}_{2} \mathrm{CrAl}$ in four different phases: (a) Bulk, (b) $\alpha$-Heuslerene, (c) $\beta$-Heuslerene, and (d) $\Upsilon$ Heuslerene

\subsubsection{Phase stability diagrams}

The phase diagram of any compound considers all possible formation by comparing to the Gibbs energies. Fig. 4 displays the phase diagram of the bulk $\mathrm{Co}_{2} \mathrm{CrAl}$ based on the following formula for its Gibbs energy:

$$
2 \mu_{\mathrm{Co}}+\mu_{\mathrm{Cr}}+\mu_{\mathrm{Al}}=g_{\text {Co2CrAl }}^{\text {bulk }}
$$

where $\mu_{\mathrm{Co}}, \mu_{\mathrm{Cr}}$, and $\mu_{\mathrm{Al}}$ are chemical potentials of the comprising atoms. The accessible area which satisfies this equation is considered as the allowed region and its borders are determined by the following equations:

$$
\begin{aligned}
& 1 / 2\left(\mathrm{G}_{\mathrm{Co} 2 \mathrm{CrAl}}-\mathrm{g}_{\mathrm{CrAl}}\right) \leq \Delta \mu_{\mathrm{Co}} \leq g_{\mathrm{Co}}^{\text {bulk }} \\
& \mathrm{G}_{\mathrm{Co} 2 \mathrm{CrAl}}-\mathrm{g}_{\mathrm{Co} 2 \mathrm{Al}} \leq \Delta \mu_{\mathrm{Cr}} \leq g_{\mathrm{Cr}}^{\text {bulk }} \\
& \mathrm{G}_{\mathrm{Co} 2 \mathrm{CrAl}}-\mathrm{g}_{\mathrm{Co} 2 \mathrm{Cr}} \leq \Delta \mu_{\mathrm{Al}} \leq g_{A l}^{\text {bulk }}
\end{aligned}
$$


in these relations, $\Delta \mu_{\mathrm{i}}$ is the chemical potential change of atom $\mathrm{i}$ and their ideal amounts are $\Delta \mu_{\mathrm{i}} \geq 0$ which means that changes are assumed to be between their maximum and minimum values. The minimum value of $\mu_{\mathrm{i}}$ is found where atom i departs from the compound and its maximum value refers to crystallization of atom $i$ in its bulk form with the Gibbs energy of $g_{i}^{b u l k}$.

Each corner in the triangle of Fig. 4a corresponds to a point where one of the atomic components of $\mathrm{Co}_{2} \mathrm{CrAl}$ leaves the structure. In other words, each corner fits the chemical potential of a specific type of atom. Therefore, the chemical potential of $\mathrm{Al}$ atom has been fixed and over the whole range of changes in other chemical potentials, the free Gibbs energies of a variety of possible combinations of $\mathrm{Co}, \mathrm{Cr}$, and $\mathrm{Al}$ has been plotted and they are all linear. Some triangles may emerge from the intersection of these lines but one of them, which is marked as a hachured area, illustrates the region where the three atoms can be simultaneously present in $\mathrm{Co}_{2} \mathrm{CrAl}$ with a space group of $\boldsymbol{F} \boldsymbol{m} \overline{\mathbf{3}} \boldsymbol{m}$. The phase diagrams of all three Heuslerene phases are compared is Fig. $4 \mathrm{~b}$ by calculating the surface free energies of these $2 \mathrm{D}$ structures through the following equation:

$$
\gamma_{\mathrm{i}}\left(\mathrm{T}, \mathrm{P}_{\mathrm{i}}\right)=\left(\mathrm{G}^{\text {slab }}-\sum_{i} N_{i} \mu_{i}\right)
$$

in which, $\mathrm{G}^{\text {slab }}$ is the surface Gibbs energy. $\mu_{\mathrm{i}}$ and $\mathrm{N}_{\mathrm{i}}$ are chemical potential and number of atoms in supercell. $\mathrm{P}_{\mathrm{i}}$ and $\mathrm{T}$ are also the partial pressure and temperatures of the components, respectively. For our 2D systems, this equation can be rephrased as below:

$\gamma_{i}\left(T, P_{i}\right)=\left(G^{\text {slab }}-\mu_{C_{0}} N_{C_{0}}-\mu_{C r} N_{C r}-\mu_{A I} N_{A I}\right) / 2 A$

in which, the factor 2 in the denominator of the right side is because of the two similar surfaces on both sides. The accessible region in this case can be found from equations 4-6. All 2D phases of $\mathrm{Co}_{2} \mathrm{CrAl}$ in Fig. $4 \mathrm{~b}$ lie into the allowed area of stability and their biggest and smallest area are observed in the $\Upsilon$ and $\alpha$ phases, respectively. Therefore, it is expected for all these Heuslerenes of $\mathrm{Co}_{2} \mathrm{CrAl}$ to be synthesized in the lab under suitable conditions. 


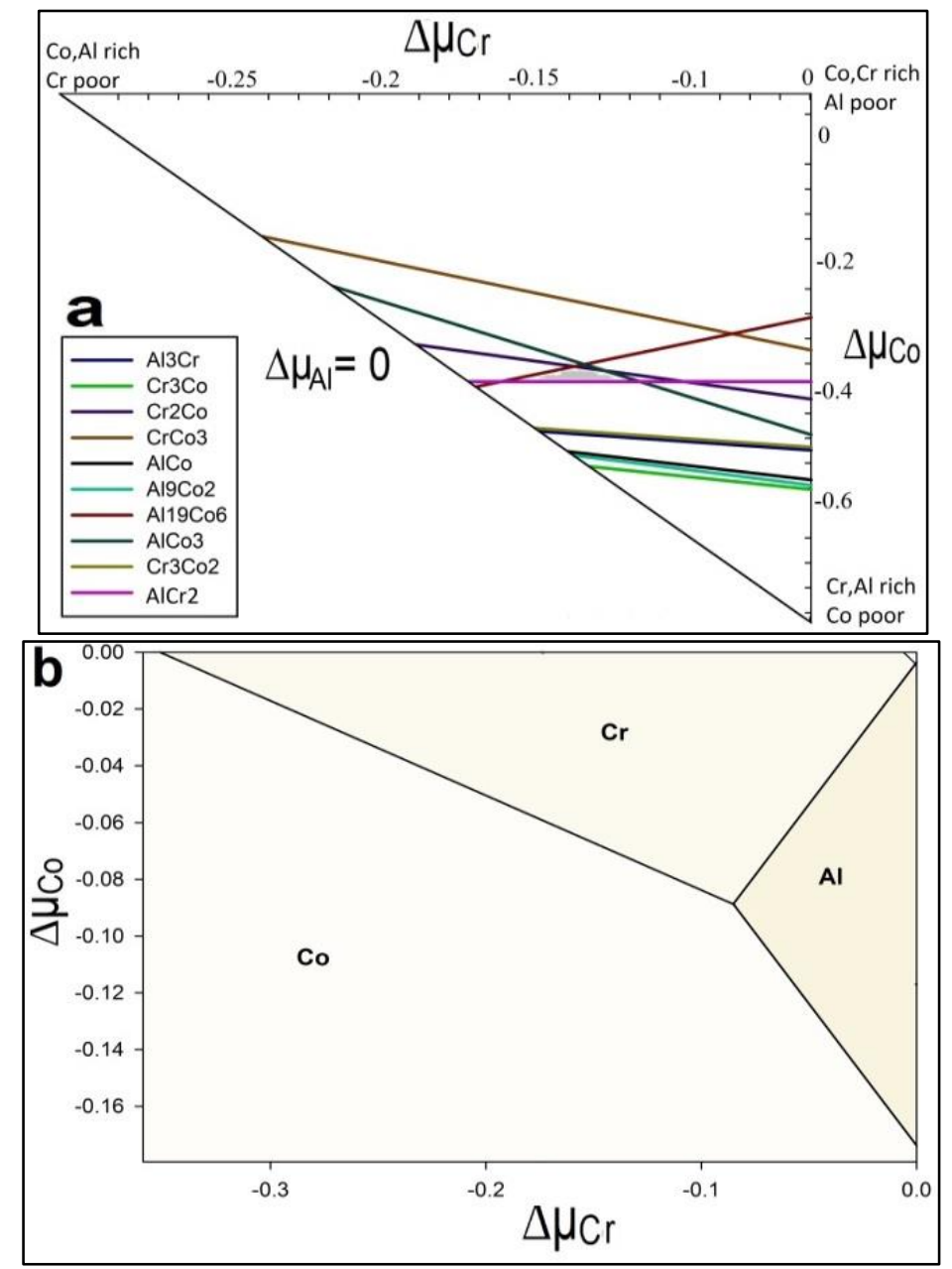

Fig. 4. The stability phase diagrams of $\mathrm{Co}_{2} \mathrm{CrAl}$ in the (a) Bulk, and (b) Heuslerene phases. Chemical changes are expressed in Rydberg unit.

\subsection{Electronic properties}

The DOS curves of the above mentioned phases of $\mathrm{Co}_{2} \mathrm{CrAl}$ for both up and down spin distributions are illustrated in Fig. 5(a-d). Fig. 5a shows that in bulk $\mathrm{Co}_{2} \mathrm{CrAl}$, the spin-up electronic states have overlapped over whole positive and negative energies and cut the Fermi level, suggesting a strong metallic nature. However, for the spin-down channel, a p-type semiconductor behavior is observed where the values of the energy gap and spin-flip gap are $0.64 \mathrm{eV}$ and $0.14 \mathrm{eV}$, respectively. Consequently, the bulk phase of $\mathrm{Co}_{2} \mathrm{CrAl}$ is a half-metal with the magnetic moment of $3 \mu_{\mathrm{B}}$ and $100 \%$ spin polarization at the Fermi level which makes it applicable in spintronics and spin injection applications. In Fig. $5(\mathrm{~b}-\mathrm{f})$, the electronic DOS of the 2D phases for the majority and minority of spins are plotted using GGA, GGA+U, and GGA+U+mbJ approximations. It is evident that comparing to the bulk, the DOS curves of all Heuslerene phases are 
more serrated which is due to their nano-scale entity and the important role of free electrons on their surfaces. The $\alpha$ and $\Upsilon$ phases seem to be metal because of both spin directions in all imposed approximations. However, they exhibit the most anisotropy in the GGA+U+mbJ approximation. Salient is the $\beta$ phase which exhibits different magnetic properties for different approximations. For the $-3 \mathrm{eV}$ to $2 \mathrm{eV}$ region, it is magnetically anisotropic in GGA while isotropic nature is achieved in GGA+U. At the Fermi level, however, it is extremely anisotropic in $\mathrm{GGA}+\mathrm{U}+\mathrm{mbJ}$. In the last case, the $\beta$ phase is a p-type semiconductor with a $0.27 \mathrm{eV}$ energy gap for spin-up. But for spin- down, a strong metal with a continuous spectrum of states is observed.

Furthermore, the electronic $\mathrm{BS}$ of the aforementioned phases of $\mathrm{Co}_{2} \mathrm{CrAl}$ have been calculated and plotted in Fig. 6. As can be seen for the minority of spins, the bulk phase is a p-type semiconductor with the direct gap of $0.64 \mathrm{eV}$ around the $\Gamma$ point which is compatible with the DOS results and other reports. However, for the majority of spins, high slope is observed in the conduction bands with the energy of more than $1 \mathrm{eV}$ which is a testament to the enormous mobility of the related electrons. For $\beta$ case, two spin-up electronic bands meet the Fermi level, as expected, and an indirect band gap appears within the spindown bands. Here, the maximum valance band and minimum conduction band are placed at $\Gamma$ and $\mathrm{K}$ points, respectively. The large gradient of the electronic states is remarkable in the conduction domain and hence, a huge degree of electronic mobility can be inferred. The difference between the BS profiles of the up and down spin channels means that the thermodynamic behavior of this Heuslerene compound can be adjusted by an external field.
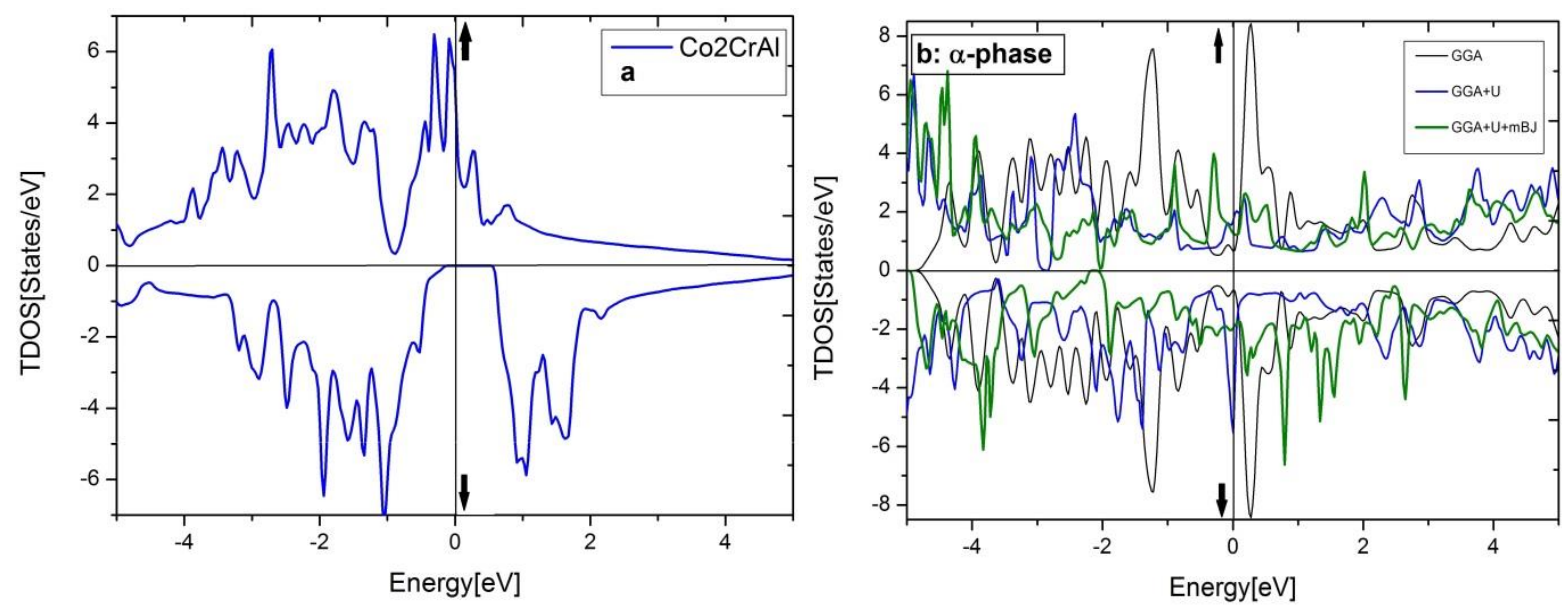

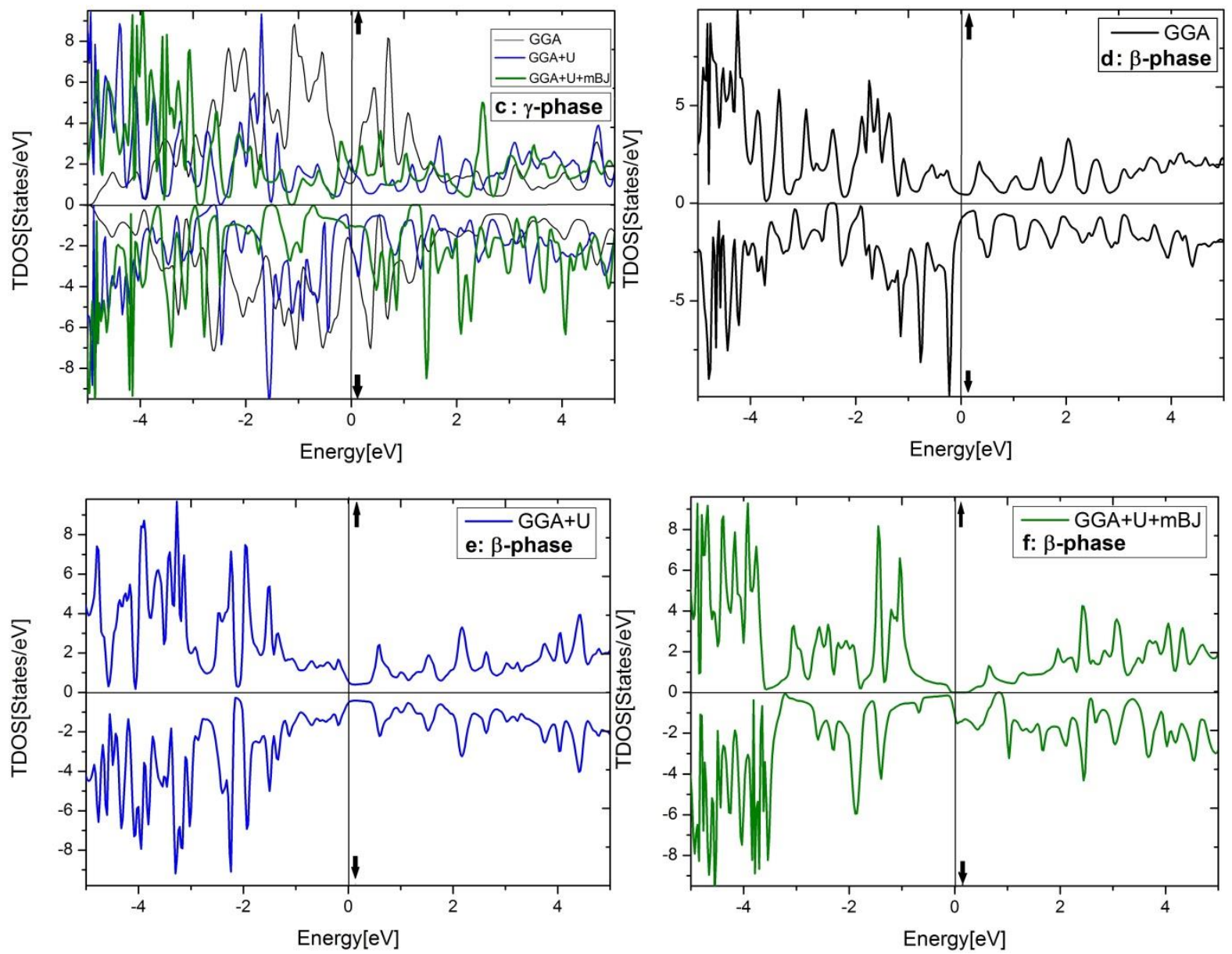

Fig. 5. The total DOS of four phases of $\mathrm{Co}_{2} \mathrm{CrAl}$ for three different approximations: (a) Bulk phase, (b) $\alpha$ phase, (c) $\Upsilon$ phase, (d) $\beta$ phase by GGA, (e) $\beta$ phase by GGA+U, and (f) $\beta$ phase by GGA+U+mbJ
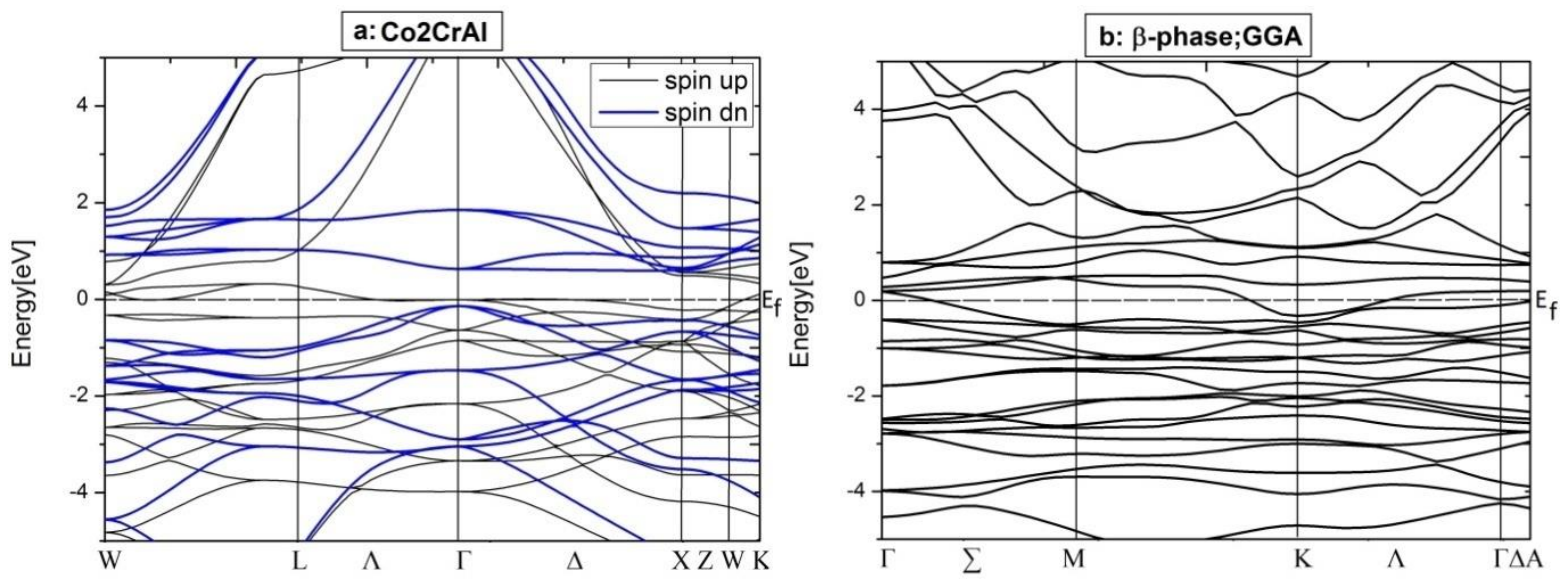

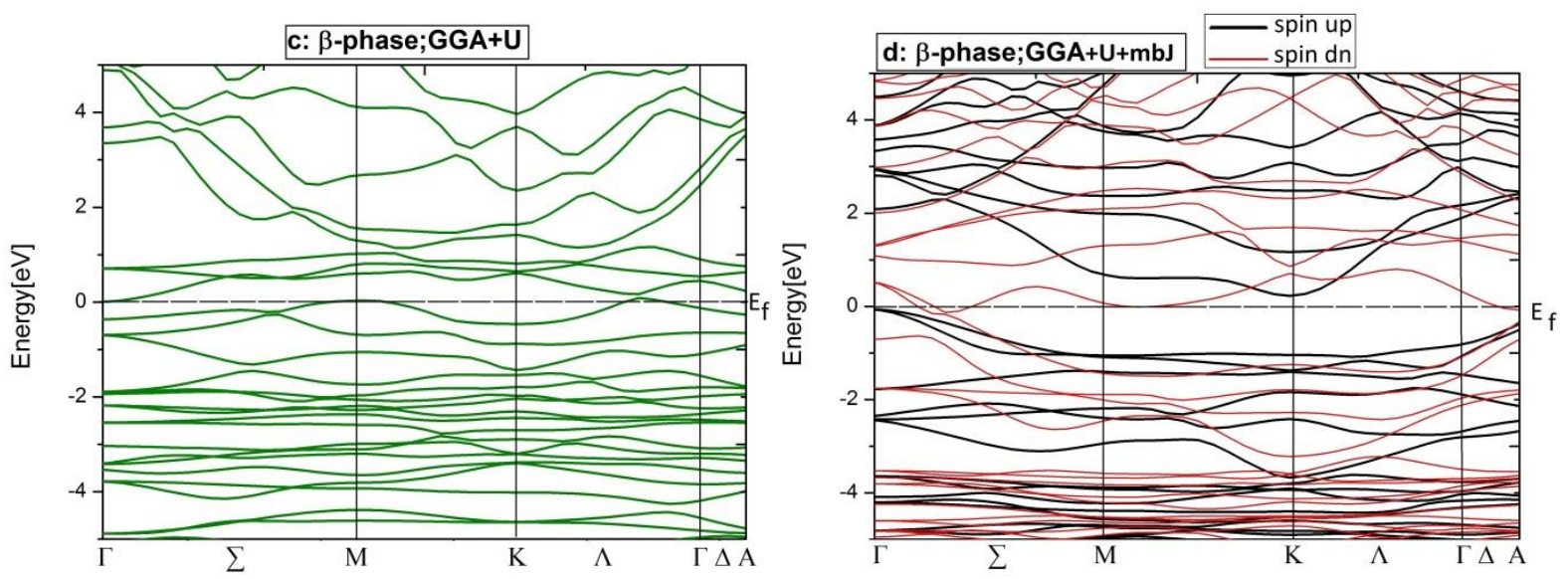

Fig. 6. Electronic BS for two spin distributions of just two phases of $\mathrm{Co}_{2} \mathrm{CrAl}$ : (a) Bulk phase, (b) $\beta$ phase by GGA, (c) $\beta$ phase by GGA $+\mathrm{U}$, and (d) $\beta$ phase by GGA $+\mathrm{U}+\mathrm{mbJ}$

In Fig. 7, an electron density diagrams are plotted on the Heuslerene $\mathrm{Co}_{2} \mathrm{CrAl}$ plate in three phases, $\alpha, \beta$ and $\Upsilon$. It is observed that at the surface of these three structures, $\mathrm{Al}, \mathrm{Cr}$ and $\mathrm{Co}$ atoms are present, respectively. The presence of electron density and dangling bonds on the surface of films and twodimensional structures gives rise to new physical properties in them. Comparison of these figures shows that the highest presence of electron density occurred at the $\alpha$ phase, and the lowest at $\beta$ case. The magnitude of the electron density in the $\alpha$ phase relative to $\beta$ one is of the order of ten. The increase in electron density at the $\alpha$ and $\Upsilon$ phases have caused them to exhibit strong metallic properties. On the other hand, the presence of $\mathrm{Cr}$ atoms in the surface of the $\beta$ phase causes more polarization of the electron density than the other two phases, and that this electron density is due to half-full $d$-orbitals that have given a magnetic treat to the electronic properties of this phase. As we saw in Table 1, the magnetic moment of this phase is an integer one. 


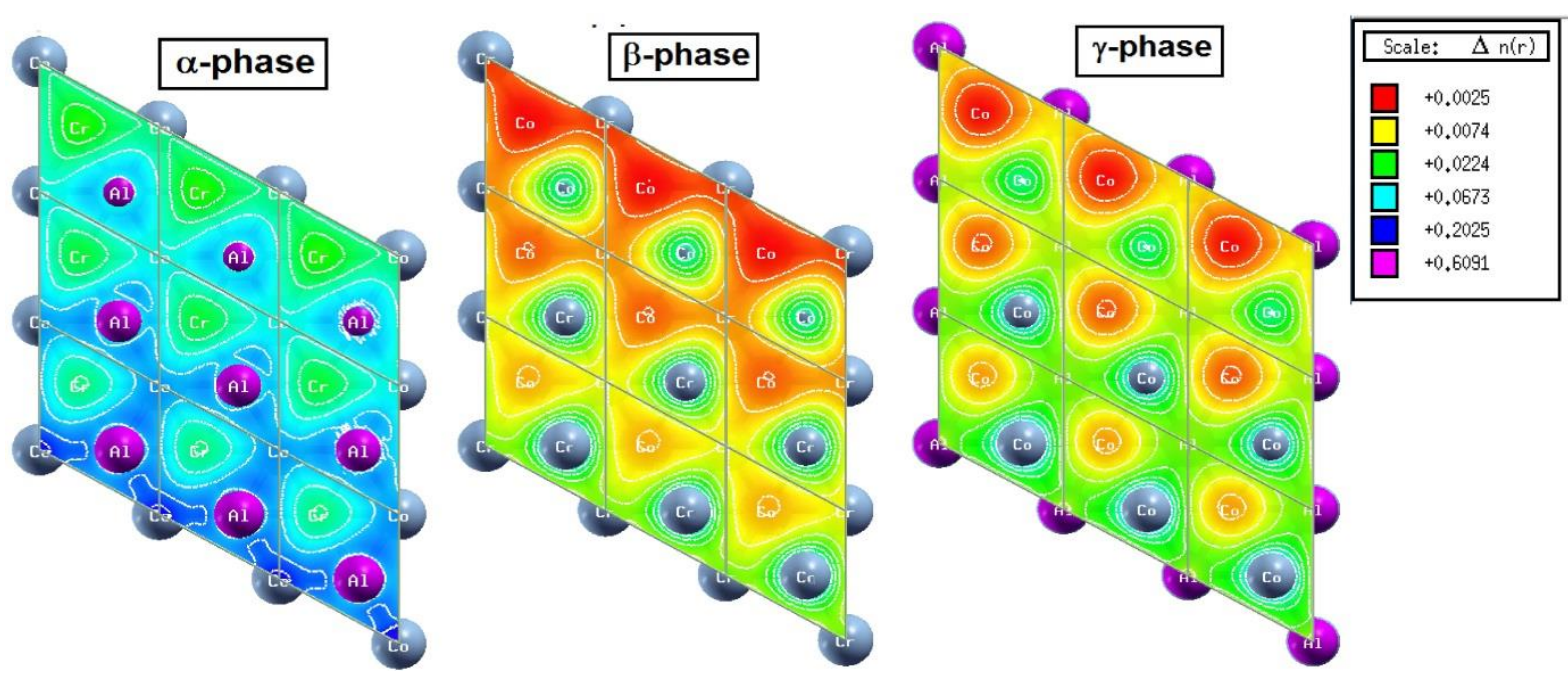

Fig. 7. Three Heuslerene monolayers of $\mathrm{Co}_{2} \mathrm{CrAl}$, named $\alpha, \beta$ and $\Upsilon$. The difference arises from the termination of the surface atoms.

\section{Conclusion}

The F.C.C structure of the Heusleres provides a compact arrangement of atoms along their [111] direction and hence, the thin films and 2D structures extracted by cutting the bulk form in this direction may be of high compactness and stability. In this work, Heuslerenes were expanded to the novel 2D structures by introducing a new member based on $\mathrm{Co}_{2} \mathrm{CrAl}$ and dealing with its expected properties in details. The crystal elastic constants for the bulk structure are obtained using the method proposed by Jamal without considering the Hubbard $\mathrm{U}$ parameter $(\mathrm{GGA}+\mathrm{U})$ in the Wien2K framework. Phonon dispersion curves were also calculated using a phonopy package.

The structural stability was estimated by the amount of the elastic constants. Also, thermodynamic characteristics were evaluated by Hill scheme as an intermediate to the Voigt and Reuss evaluations Our ab initio results are in good agreement with previous theoretical calculations. The value of Poisson's ratio confirmed the ionic domain bonds which is compatible with our deduction from the positive sign of Cauchy pressure. Besides, the ductile nature was inferred from the percentage of $\left(\frac{B_{H}}{S_{H}}\right)$ ratio in bulk $\mathrm{Co}_{2} \mathrm{CrAl}$ which was $214 \%$. The values of Vickers Hardness, based on Chen's and Tian's equations, were positive and almost close to each other. The directional values of Young's moduli revealed the isotropic nature of all three Heuslerene phases of $\mathrm{Co}_{2} \mathrm{CrAl}$ along the $\boldsymbol{x}$ and $\boldsymbol{y}$ directions. Moreover, moving from the bulk towards the surface decreased the hardness. Comparing to other $\mathrm{Co}_{2} \mathrm{CrAl}$ Heuslerene phases, the elastic constants $\left(C_{11}\right.$ and $C_{12}$ and $\left.C_{66}\right)$ of $\mathrm{Co}_{2} \mathrm{CrAl}-\mathrm{Al}$ were larger and hence, it was mechanically 
stronger. The positive value of all frequency branches of phonon distribution diagrams implied that all phases of $\mathrm{Co} 2 \mathrm{CrAl}$ were dynamically stable. Also, all 2D predicted forms of $\mathrm{Co}_{2} \mathrm{CrAl}$ were strongly stable against the lattice vibrations.

According to our phase diagram calculations of bulk $\mathrm{Co}_{2} \mathrm{CrAl}$, a hachured area illustrated the region where all three atoms could be simultaneously placed in $\mathrm{Co}_{2} \mathrm{CrAl}$ with a space group of $\boldsymbol{F} \boldsymbol{m} \overline{\mathbf{3}} \boldsymbol{m}$. All 2D phases of $\mathrm{Co}_{2} \mathrm{CrAl}$ lied into the allowed area of stability and the biggest and smallest area belonged to the $\Upsilon$ and $\alpha$ phase, respectively.

In addition, the half-metallic nature of bulk phase of $\mathrm{Co}_{2} \mathrm{CrAl}$ was demonstrated by electronic investigations for which the value of magnetic moment and the percentage of spin polarization at the Fermi level was $3 \mu_{\mathrm{B}}$ and $100 \%$, respectively and hence, it could be employed for spintronics and spin injection applications. The $\alpha$ and $\Upsilon$ phases seemed to be metal in both spin directions and all approximations but the most anisotropy was exhibited in the GGA+U+mbJ approximation. Finally, Salient was the $\beta$ phase which exhibited different magnetic properties in different approximations, such that is magnetically anisotropic in $-3 \mathrm{eV}$ to $2 \mathrm{eV}$ by GGA, isotropic. For the $-3 \mathrm{eV}$ to $2 \mathrm{eV}$ region, it was magnetically anisotropic in GGA while isotropic nature was achieved in GGA+U. At the Fermi level, however, it was extremely anisotropic in GGA $+\mathrm{U}+\mathrm{mbJ}$. Also, the $\beta$ phase was a p-type semiconductor with a $0.27 \mathrm{eV}$ energy gap for spin-up. But for spin- down, a strong metal with a continuous spectrum of states was observed. 


\section{Refrences}

[1] A. Amudhavalli, R. Rajeswarapalanichamy, K. Iyakutti (2018) Computational Materials Science, 148, 87-103.

[2] A. Amudhavalli, R. Rajeswarapalanichamy, K. Iyakutti (2017) Journal of Alloys and Compounds, 708, 1216-1233.

[3] P. J. Webster, K. R. A. Ziebeck (1973) Journal of Physics and Chemistry of Solids, 34(10), 1647-1654.

[4] J. Kübler, A. R. William, C. B. Sommers (1983) Physical Review B, 28(4), 1745.

[5] R. A. De Groot, F. M. Mueller (1983) Physical Review Letters, 50(25), 2024.

[6] A. Hirohata, , et al. (2020) Journal of Magnetism and Magnetic Materials, 166711.

[7] S. Ishida, S. Akazawa, Y. Kubo, J. Ishida (1982) Journal of Physics F: Metal Physics, 12(6), 1111.

[8] B. Y. Tong, L. J. Sham (1966) Physical Review, 144(1), 1.

[9] L. Hedin, B. I. Lundqvist (1971) Explicit local exchange-correlation potentials. Journal of Physics C: Solid state physics, 4(14), 2064.

[10] U. von Barth, L. Hedin (1972) Journal of Physics C: Solid State Physics, 5(13), 1629.

[11] S. H. Vosko, L. Wilk, M. Nusair (1980) Canadian Journal of physics, 58(8), 1200-1211.

[12] S. Ishida, S. Fujii, S. Kashiwagi, S. Asano (1995) Journal of the Physical Society of Japan, 64(6), 2152-2157.

[13] S. Ishida, S. Kashiwagi, S. Fujii, S. Asano (1995) Physica B: Condensed Matter, 210(2), 140-148.

[14] P. Mohn, P. Blaha, K. Schwarz (1995) Journal of magnetism and magnetic materials, 140, 183-184.

[15] I. Galanakis, P. H. Dederichs, N. Papanikolaou (2002) Physical Review B, 66(17), 174429.

[16] S. Picozzi, A. Continenza, A. J. Freeman (2002) Physical Review B, 66(9), 094421.

[17] Y. Miura, K. Nagao, M. Shirai, (2004) Physical Review B, 69(14), 144413. [18] V. N. Antonov, H. A. Dürr, Y. Kucherenko, L. V. Bekenov, A. N. Yaresko (2005) Physical Review B, 72(5), 054441.

[19] H. L. Huang, J. C. Tung, G. Y. Guo (2015) Physical Review B, 91(13), 134409.

[20] J. Kübler, G. H. Fecher, C. Felser (2007) Physical Review B, 76(2), 024414.

[21] Q. H. Wang, K. Kalantar-Zadeh, A. Kis (2012) Nature nanotechnology, 7(11), 699-712. 
[22] G. Zhang, Y. W. Zhang (2017) Journal of Materials Chemistry C, 5(31), 7684-7698.

[23] K. Liu, J. Wu (2016) Journal of Materials Research, 31(7), 832-844.

[24] F. Schedin, et al. (2007) Nature materials, 6(9), 652-655.

[25] P. Blake, et al. (2008) Nano letters, 8(6), 1704-1708.

[26] M. F. El-Kady, V. Strong, S. Dubin, R. B. Kaner (2012) Science, 335(6074), 1326-1330.

[27] J. D. Fowler, M. J. Allen, V. C. Tung, Y. Yang, R. B. Kaner, B. H. Weiller (2009) ACS nano, 3(2), 301-306.

[28] S. H. Bae, Y. Lee, B. K. Sharma, H. J. Lee, J. H. Kim, J. H. Ahn (2013) Carbon, 51, 236-242.

[29] F. Schwierz (2010) Nature nanotechnology, 5(7), 487.

[30] A. Sakhaee-Pour, M. T. Ahmadian, A. Vafai (2008) Solid State Communications, 147(7-8), 336-340.

[31] A. Acun, et al. (2015) Journal of physics: Condensed matter, 27(44), 443002.

[32] V. O. Ozcelik, D. Kecik, E. Durgun, S. Ciraci (2015)The Journal of Physical Chemistry C, 119(1), 845-853.

[33] D. Laniel, G. Geneste, G. Weck, M. Mezouar, P. Loubeyre (2019) Physical review letters, 122(6), 066001.

[34] A. V. Penumatcha, R. B. Salazar, J. Appenzeller (2015) Nature communications, 6(1), 1-9.

[35] G. Pizzi, M. Gibertini, E. Dib, N. Marzari, G. Iannaccone, G. Fiori (2016) Nature communications, 7, 12585.

[36] P. Ares, J. J. Palacios, G. Abellán, J. Gómez-Herrero, F. Zamora (2018) Advanced Materials, 30(2), 1703771.

[37] J. Qiao, X. Kong, Z. X. Hu, F. Yang, W. Ji (2014) Nature communications, $5(1), 1-7$.

[38] F. Xia, H. Wang, Y. Jia (2014) Nature communications, 5(1), 1-6.

[39] J. O. Island, G. A. Steele, H. S. van der Zant, A. Castellanos-Gomez (2015) 2D Materials, 2(1), 011002.

[40] Y. Huang, et al. (2016) Chemistry of Materials, 28(22), 8330-8339.

[41] C. Tan, X. Cao, X. J. Wu, Q. He, J. Yang, X. Zhang,...\& M. Sindoro (2017) Chemical reviews, 117(9), 6225-6331.

[42] J. S. Lee, et al. (2018) Science, 362(6416), 817-821.

[43] H. Wang, C. Li, P. Fang, Z. Zhang, J. Z. Zhang (2018) Chemical Society Reviews, 47(16), 6101-6127.

[44] X. Duan, C. Wang, A. Pan, R. Yu, X. Duan (2015) Chemical Society Reviews, 44(24), 8859-8876.

[45] S. Manzeli, D. Ovchinnikov, D. Pasquier, O. V. Yazyev, A. Kis (2017) Nature Reviews Materials, 2(8), 17033. 
[46] J. Chen, G. H. Ryu, S. Sinha, J. H. Warner (2019) Atomic structure and dynamics of defects and grain boundaries in $2 \mathrm{D} \mathrm{Pd} 2 \mathrm{Se} 3$ monolayers. ACS nano, 13(7), 8256-8264.

[47] A. Boochani, et al. (2019) Journal of Materials Chemistry C, 7(43), 1355913572.

[48] A. Boochani, B. Nowrozi, J. Khodadadi, S. Solaymani, S. Jalali-Asadabadi (2017) The Journal of Physical Chemistry C, 121(7), 3978-3986.

[49] P. Blaha, K. Schwarz, G. K. H. Madsen, D. Kuasnicke, J. Luitz, Introduction to WIEN2K. An Augmented plane wane plus local orbitals program for calculating crystal properties (Vienna University of Technology, Vienna, Austria, 2001).

[50] E. Sjöstedt, L. Nordström, D. J. Singh (2000) Solid state communications, 114(1), 15-20.

[51] J. P. Perdew, et al. (2007) arXiv preprint arXiv:0707.2088.

[52] A. I. Liechtenstein, V. I. Anisimov, J. Zaanen, (1995) Physical Review B, 52(8), R5467.

[53] P. Giannozzi, et al., (2009) J.Phys.:Condens.Matter 21, 395502.

[54] P Giannozzi, et. al., (2017) J.Phys.:Condens.Matter 29, 465901.

[55] M. Jamal, M. Bilal, I. Ahmad, S. Jalali-Asadabadi (2018) Journal of Alloys and Compounds, 735, 569-579.

[56] M. Jamal, S. J. Asadabadi, I. Ahmad, H. R. Aliabad (2014) Computational Materials Science, 95, 592-599.

[57] Y. Wang, J. Cheng, M. Behtash, W. Tang, J. Luo, K. Yang (2018) Physical Chemistry Chemical Physics, 20(27), 18515-18527.

[58] M. Ilkhani, A. Boochani, M. Amiri, M. Asshabi, D. P. Rai (2020) Solid State Communications, 308, 113838.

[59] V. I. Anisimov, J. Zaanen, O. K. Andersen (2991) Physical Review B, 44(3), 943.

[60] P. Blaha, K. Schwarz, G. K. Madsen, D. Kvasnicka, J. Luitz (2001) J. wien2k. An augmented plane wave+ local orbitals program for calculating crystal properties.

[61] M. Jamal, N. KamaliSarvestani, A. Yazdani and A. H. Reshak (2014) RSC Adv. 4, 57903.

[62] M. Jamal, S. JalaliAsadabadi, Iftikhar Ahmad, H.A. Rahnamaye Aliabad (2014) Computational Materials Science 95, 592.

[63] M. Jamal, M. Bilal, Iftikhar Ahmad, S. Jalali-Asadabadi (2018) Journal of Alloys and Compounds, 735, 569.

[64] IRelast2D package is provided by M. Jamal. It will be as part of the commercial code WIEN2K, http://www.wien2k.at/.

[65] P. Blaha, K. Schwarz, G. K. H. Madesen, D. Kvasnicka, J, Luitz, WIEN2K, An Augmented Plane Wave + Local Orbitals Program for Calculating Crystal Proper-ties, Karlheinz Schwarz, Techn; Universitaetwien:Wein, Austria, 2001. 
[66] H. D. Espinosa, R. A. Bernal, and T. Filleter (2012) Small. 8, 3233.

[67] A. Yildirim, H. Koc and E. Deligoz (2012) Chin. Phys. B, 21, 037101.

[68] M. G. Brik, (2010) J. Phys. Chem. Solids, 71, 1435.

[69] X.Q. Chen, H. Niu, D. Li, Y. Li (2011) Intermetallics, 19, 1275.

[70] Y. Tian, B. Xu, Z. Zhao, (2012) Inte. J. Refract. Metal. Hard Mater, 33, 93.

[71] S. Wu, G. H. Fecher, S. S. Naghavi, C. Felser (2019) J. Appl. Phys. 125, 082523.

[72] H. Salehi, M. Halvaei, P. Amiri, (2018) J. R. Many-body Systems, 8, 69.

[73] P. Blaha, K. Schwarz, P. Sorantin, S. B. Trickey (1990) Comput. Phys. Commun. 59, 399.

[74] J. Perdew, J. A. Chevary, S. H. Vosko, K. A. Jackson, M. R. Pederson, D. J. Singh, C. Fiolhais (1992) Phys. Rev. B, 46, 6671.

[75] F. Mouhat, F.X. Coudert (2014) Phys. Rev. B, 90, 224104.

[76] S.H. Zhang, R.F. Zhang, (1017) Computer Physics Communications, 220, 403.

[77] Y. Chen, X. An, X. Liao, (2017) Appl. Phys. Rev. 4, 031104.

[78] B. Wu, A. Heidelberg, and J. J. Boland, (2005) Nat. Mater. 4, 525.

[79] C. Herring and J. K. Galt (1952) Phys. Rev. 85, 1060.

[80] S. S. Brenner (1956) J. Appl. Phys. 27, 1484.

[81] R. E. Miller and V. B. Shenoy (2000) Nanotechnology, 11, 139.

[82] T. Zhu and J. Li (2010) Prog.Mater.Sci. 55, 710.

[83] J. R. Greer and J. T. M. De Hosson (2011) Prog. Mater.Sci. 56, 654. 